\title{
Oligodeoxynucleotide IMT504 induces a marked recovery in a streptozotocin-induced model of diabetes in rats: correlation with an early increase in the expression of nestin and neurogenin 3 progenitor cell markers
}

\author{
M. S. Bianchi • A. Hernando-Insúa • N. A. Chasseing • \\ J. M. Rodríguez • F. Elías • N. Lago • J. Zorzopulos • \\ C. Libertun • A. D. Montaner • V. A. Lux-Lantos
}

Received: 26 August 2009 / Accepted: 13 January 2010/Published online: 11 March 2010

(C) Springer-Verlag 2010

\begin{abstract}
Aims/hypothesis IMT504 is an oligonucleotide that promotes tissue repair in bone injury and neuropathic pain models by stimulating progenitor cells. Here we evaluated the effect of IMT504 on the recovery of islet function in a streptozotocin (STZ)-induced model of diabetes in the rat.

Methods Male Sprague-Dawley rats were injected with STZ (60 mg/kg, i.p., day 1) or citrate buffer (Control). Animals with glycaemia between 11 and $20 \mathrm{mmol} / \mathrm{l}$ on day 4 were injected with IMT504 $(4 \mathrm{mg} /$ animal in saline, s.c.,
\end{abstract}

Electronic supplementary material The online version of this article (doi:10.1007/s00125-010-1694-z) contains supplementary material, which is available to authorised users.

M. S. Bianchi $\cdot$ N. A. Chasseing $\cdot$ C. Libertun · V. A. Lux-Lantos Institute of Biology and Experimental Medicine (IBYME-CONICET), Buenos Aires, Argentina

A. Hernando-Insúa · J. Zorzopulos · A. D. Montaner Immunotech,

Buenos Aires, Argentina

A. Hernando-Insúa $\cdot$ J. M. Rodríguez $\cdot$ F. Elías $\cdot$ A. D. Montaner
Fundación Pablo Cassará,
Buenos Aires, Argentina

N. Lago

Gema Biotech,

Buenos Aires, Argentina

M. S. Bianchi - N. A. Chasseing $\cdot$ C. Libertun •

V. A. Lux-Lantos $(\bowtie)$

V. de Obligado 2490, (C1428ADN),

Buenos Aires, Argentina

e-mail: vlux@dna.uba.ar
STZ-IMT504) or with saline (STZ-Saline) for 10 days. Glycaemia and water and food intake were recorded for 33 days. Intraperitoneal glucose tolerance tests (IPGTTs) were performed on day 30 . On day 35 , overnight-fasted animals were killed and blood samples and pancreases collected for hormonal and histological studies. A second group of STZ-IMT504 rats was killed, together with Control and STZ-Saline rats, after two consecutive days of blood glucose decreases after the beginning of IMT504 treatment. Pancreases were collected and proliferating cell nuclear antigen (PCNA), nestin and neurogenin 3 (NGN3) detected by immunohistochemistry.

Results IMT504 greatly improved blood glucose and food and water intakes in STZ-IMT504 rats by day 8, as well as IPGTTs on day 30. Significant increases in islet number and beta cell content were observed in STZ-IMT504 rats (day 33). Furthermore, after two to five IMT504 injections, blood glucose decreased, and an increase in pancreatic nestin (mainly in endothelial cells), PCNA and NGN3 production (in islets) was observed in STZ-IMT504 rats. Conclusions/interpretation IMT504 induced a marked recovery of STZ-induced diabetes that correlated with early production of progenitor cell markers, such as nestin and NGN3.

Keywords Glycaemia - IMT504 - Islet recovery · Nestin . NGN3 · Oligodeoxynucleotide · PCNA · Progenitors · STZ
Abbreviations
BW
DAB 3,3'-Diaminobenzidine
HOMA-beta HOMA of beta cell function 
HOMA-IR HOMA of insulin resistance

IPGTT Intraperitoneal glucose tolerance test

NGN3 Neurogenin 3

ODN Oligodeoxynucleotide

PCNA Proliferating cell nuclear antigen

PPCM Pancreatic progenitor cell marker

STZ Streptozotocin

\section{Introduction}

In type 1 diabetes, immunity and inflammation play an important role in beta cell destruction. Extensive efforts have been directed towards the development of immunomodulatory treatments for maintaining and restoring beta cell mass $[1,2]$.

Immunomodulatory oligodeoxynucleotides (ODNs) are synthetic molecules that stimulate several immune cells, causing activation, proliferation and immunoglobulin secretion [3]. ODNs active in humans are: (1) CpG ODNs, with at least one active site bearing an unmethylated $\mathrm{CpG}$ (a single strand cytosine-guanine dinucleotide with 5'-3' direction) in a given context [4], and (2) PyNTTTTGT ODNs with at least one active site bearing the sequence PyNTTTTGT, in which Py is $\mathrm{C}$ or T and $\mathrm{N}$ is $\mathrm{A}, \mathrm{T}, \mathrm{C}$ or $\mathrm{G}$ [3], this being IMT504, a prototype of this type. Both types have been assayed as adjuvants in vaccines and in cancer and allergy therapies. Whereas $\mathrm{CpG}$ ODNs have been evaluated in autoimmune diseases [1], no data are available regarding IMT504. Interestingly, while IMT504 is a potent stimulator of mesenchymal stem cells (MSCs), CpG ODNs are not [5]. We have shown that IMT504 is useful for tissue repair in a bone injury model [5], presumably through expansion of the MSC pool and/or modifications in the immunochemical microenvironment at the site of damage. These results prompted us to investigate the effects of IMT504 on glucose homeostasis and pancreatic progenitor cell markers (PPCMs) in a streptozotocin (STZ)-induced model of diabetes in the rat.

\section{Methods}

IMT504 (Oligos Etc., Wilsonville, OR, USA) was treated as described [5], its sequence being 5'-TCATCATTTTGT CATTTTGTCATT-3'.

Male Sprague-Dawley rats (Institute of Biology and Experimental Medicine [IBYME] colony, 180-220 g) were injected i.p. with STZ (60 mg/kg body weight [BW], freshly dissolved in $0.05 \mathrm{mmol} / \mathrm{l}$ sodium citrate buffer, $\mathrm{pH} 4.5$ ) at 12:00 hours (day 1). At this dose, STZ acts through uptake by the glucose transporter GLUT2 and induces beta cell toxicity. Controls received citrate buffer. Animals were given free access to laboratory chow and water. Studies were performed according to institutional protocols for animal use (IBYME-CONICET and the National Institutes of Health [NIH]).

First design

As from day 4, STZ animals (glycaemia: 11-20 mmol/l) and Controls were s.c. injected with saline (STZ-Saline and Control-Saline) or with IMT504 (4 mg in $0.2 \mathrm{ml}$ saline daily) for ten consecutive days (STZ-IMT504 and ControlIMT504). Glycaemia was monitored every 4 days until day 33 , at 12:00 hours, in non-fasted animals, in tail vein blood samples using a blood glucose monitoring system (LifeScan, Milpitas, CA, USA).

For intraperitoneal glucose tolerance tests (IPGTTs), on day 30 overnight-fasted animals were injected i.p. with $2 \mathrm{~g} / \mathrm{kg}$ BW glucose and glycaemia was measured at 0, 30, 60, 90 and $120 \mathrm{~min}$.

Food and water intake were recorded in the morning every 2-3 days. BW was measured and food efficiency calculated.

HOMA of insulin resistance (HOMA-IR) and HOMA of beta cell function (HOMA-beta) indices were calculated as previously described [6].

On day 35, after measuring blood glucose, overnightfasted animals were decapitated, sera collected for insulin determination (RIA) and pancreases processed for histological analysis.

\section{Second design}

Starting on day 4, STZ animals (glycaemia: 11-20 mmol/l), were treated daily with IMT504, as above, and killed after two consecutive decreases in blood glucose. Control and STZ-Saline rats were killed simultaneously. Before killing, the blood glucose level in the non-fasted animals was measured and sera were kept for insulin measurement. Rats were then anaesthetised (ketamine/xylacine, 60/10 mg/kg BW, i.p.), and transcardiacally perfused with $0.9 \%$ (wt/vol.) saline followed by $4 \%$ (wt/vol.) paraformaldehyde in PBS, $\mathrm{pH}$ 7.4. Pancreases were further processed as above.

Pancreas histological analysis

First design Four sections from different regions of each pancreas ( $n=5$ per group) were used for double staining for insulin and glucagon. Glucagon: rabbit polyclonal anti-rat glucagon (sc 13091, 1:300; Santa Cruz Biotechnology, Santa Cruz, CA, USA) and alkaline phosphatase Vectastain ABCAP KIT (Vector, Burlingame, CA, USA) combined with a Vector blue AP Kit (Vector, SK-5300). Insulin: guinea pig 
anti-rat insulin (Cat ab 7842UK, 1:300; Abcam, Cambridge, MA, USA) and a Vectastain ABC-AP kit combined with a Vector red AP kit (Vector, SK-5100).

Second design In all cases antigen retrieval was performed. To determine cell proliferation, triple staining for insulin, glucagon and proliferating cell nuclear antigen (PCNA) was performed. Insulin was stained as above, glucagon was stained as above but followed by a Vectastain ABC kit and 3,3'-diaminobenzidine (DAB), and PCNA was detected with an anti-mouse PCNA antibody (MAB424, 1:200; Millipore, Billerica, MA, USA) followed by a Vectastain ABC-AP kit combined with a Vector blue AP Kit.

To localise the staining of PPCMs, double staining for insulin and nestin or neurogenin 3 (NGN3) were performed. For insulin and nestin, insulin was detected as above, and nestin was detected with mouse anti-nestin antibody (Rat-401, 1:30; Developmental Studies Hybridoma Bank, University of Iowa, Iowa City, IA, USA), followed by a Vectastain $\mathrm{ABC}$ kit and DAB. Slides were counterstained with haematoxylin. For double staining for insulin and NGN3, insulin was detected as above, and NGN3 was identified with an anti-NGN3 antibody (F25A1B3, 1:500; Developmental Studies Hybridoma Bank), followed by a Vectastain ABC-AP kit combined with a Vector blue AP Kit.

To determine the presence of angiogenesis, double staining for insulin and CD31 was performed. Insulin was detected as above, but followed by a Vectastain ABC-AP kit combined with a Vector blue AP Kit, and CD31 was detected with a goat polyclonal anti-PECAM-1 antibody (sc-1506, 1:100; Santa Cruz Biotechnologies), followed by a Vectastain $\mathrm{ABC}$ kit and DAB. Control-Saline: $n=90$ islets, $n=257$ blood vessels; STZ-Saline: $n=55$ islets, $n=$ 459 blood vessels and STZ-IMT504: $n=80$ islets, $n=648$ blood vessels.

In all cases, non-specific staining was evaluated in the absence of primary antibodies. Image analysis was performed using a Nikon Photomicroscope Eclipse 200 and Image-Pro Plus v4.5 software (Microlab, Buenos Aires, Argentina).

\section{Statistics}

Results are expressed as means \pm SEM. Differences between means were analysed by one- or two-way ANOVA, followed by a Newman-Keuls test or a Tukey HSD test for unequal $N$; for multiple determinations in the same animal, two-way ANOVA with repeated measures design was used (Statistica 8). Only for nestin-positive pancreases/ total pancreases, frequency distributions were analysed using the $\chi^{2}$ test. $p<0.05$ was considered statistically significant.

\section{Results}

First design

IMT504 induced a marked recovery of the diabetic condition in STZ-treated rats IMT504 induced a rapid decrease in glycaemia in STZ-injected animals; by day 8 , blood glucose was normalised in $90 \%$ of STZ-IMT504 rats (nine of ten; Fig. 1a). Glycaemia was not affected in Control-IMT504 rats. IMT504 greatly improved IPGTTs in STZ-IMT504 rats, without modifying glucose disposal in Control-IMT504 rats (Fig. 1b). IMT504 normalised beta cell function (HOMA-beta) and insulin resistance (HOMAIR; Fig. 1c). Histological analysis (Fig. 2a-d) showed that the insulin-positive area decreased in STZ-saline and partially recovered in STZ-IMT504 rats (Fig. 2e), as did the number of islets per pancreatic area (Fig. 2f) and the islet surface area (Fig. 2g). Glucagon-positive area was not modified by STZ or IMT504 (Fig. 2e). Control-IMT504 islets were similar to Control-Saline (Fig. 2e-g).

IMT504 greatly improved polydipsia, polyphagia and BW in STZ-IMT504 rats. A per se anorexigenic effect of IMT504 seemed to add to the decrease in food intake, as it was also observed in Control-IMT504 rats. In addition, a decrease in BW was temporarily observed in ControlIMT504 rats. Food efficiency was normalised in STZIMT504 and severely affected in STZ-Saline rats (Electronic supplementary material [ESM] Fig. 1). IMT504-induced decrease in food intake, and therefore in the challenge for insulin secretion, could participate in the recovery of islet function and blood glucose.

\section{Second design}

IMT504 induced the early production of tissue repair markers in STZ diabetic rats After two consecutive days of blood glucose decrease (two to five IMT504 doses), three of six STZ-IMT504 rats had normalised their glycaemia $(6.6 \pm 1.1 \mathrm{mmol} / \mathrm{l})$. Although the others were still hyperglycaemic $(15.0 \pm 0.4 \mathrm{mmol} / \mathrm{l})$, glycaemia had decreased from its peak. At this time STZ-Saline rats were hyperglycaemic $(24.4 \pm 1.9 \mathrm{mmol} / \mathrm{l})$. Serum insulin was similar in ControlSaline and STZ-Saline rats but significantly increased in STZIMT504 rats ( $p<0.05$ vs Control-Saline), while islet insulin had partially recovered in STZ-IMT504 rats (ESM Fig. 2).

An increase in markers of tissue repair was observed in STZ-IMT504 pancreases at this time. The percentage of PCNA-positive islets and the number of PCNA-positive nuclei per islet increased significantly in STZ-IMT504 rats compared with Control-Saline (Fig. 3a, b). In islets, PCNA was mainly positive in beta cells in all groups.

Nestin was present in $25 \%$ of Control-Saline pancreases. Those from STZ-Saline rats were nestin-negative. 

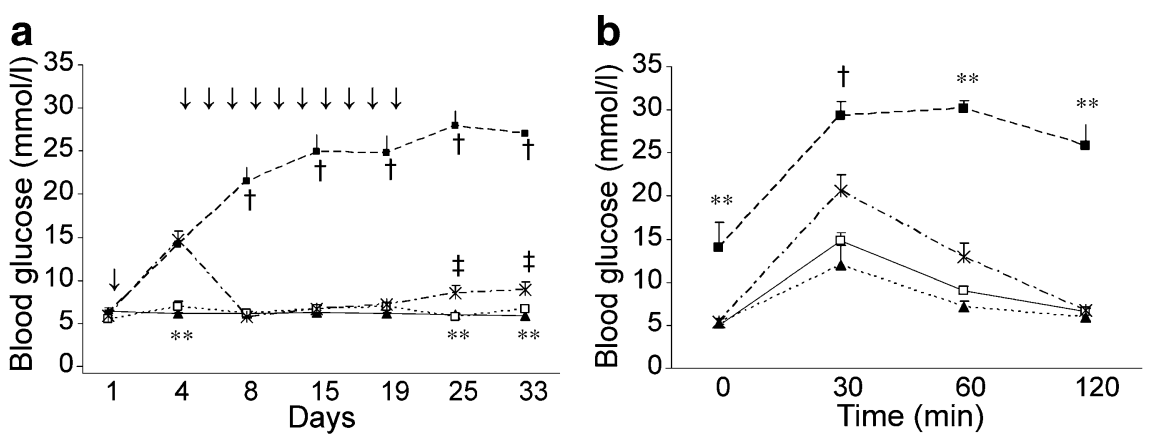

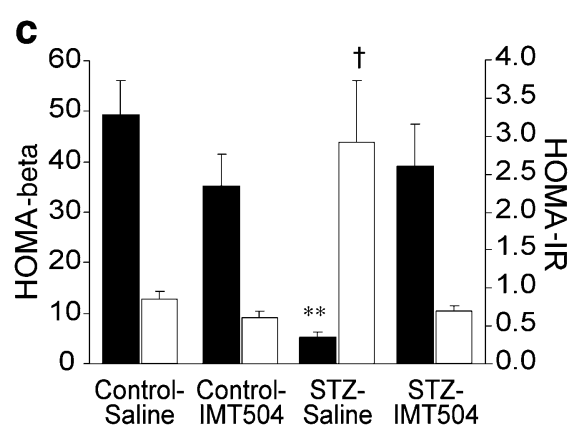

Fig. 1 IMT504 induced a marked recovery of the diabetic condition in STZ-treated rats. a Effect of IMT504 treatment on basal glucose. Control-Saline ( $n=10$, black triangles), STZ-Saline $(n=9$, black squares), STZ-IMT504 ( $n=9$, asterisks; this and the following figures show the data of the 9/10 STZ-IMT504 responding animals) and Control-IMT504 ( $n=5$, white squares) rats. Arrows indicate IMT504 treatment. Two-way ANOVA for repeated measures, interaction $p<$ 0.01 . Tukey HSD test for unequal $n$ : ${ }^{*}$ Control-Saline different from STZ-Saline and STZ-IMT504 $(p<0.01),{ }^{\dagger}$ STZ-Saline different from all $(p<0.01)$, ${ }^{\ddagger}$ STZ-IMT504 different from all $(p<0.01)$. b Effect of
IMT504 on IPGTTs. Control-Saline $(n=10)$, STZ-IMT504 $(n=9)$, STZ-Saline $(n=5)$ and Control-IMT504 $(n=5)$. Two-way ANOVA for repeated measures, interaction $p<0.01$. Tukey HSD test for unequal $n$ : **different from all $(p<0.01),{ }^{\dagger}$ different from Control-Saline and Control-IMT504 $(p<0.01)$. c Effect of IMT504 on HOMA-beta (black bars) and HOMA-IR (white bars). HOMA-beta, one-way ANOVA $p<0.01$. Tukey HSD test for unequal $n$ : **different from all $(p<0.01)$. HOMA-IR, one-way ANOVA $p<0.01$. Tukey HSD test for unequal $n:{ }^{\dagger}$ different from all $(p<0.01)$
In STZ-IMT504 rats, 67\% were nestin-positive, significantly different from STZ-Saline $\left(\chi^{2}, p<0.05\right.$; Fig. 3f). Nestin was observed in endothelial cells in pancreatic parenchyma, within a few islets, and also in pericytes, stellate and exocrine cells (Fig. $3 g-j$ ).
CD31 increased in STZ-IMT504 rats compared with Control-Saline in islets $(p<0.01)$ and blood vessels $(p<$ 0.01) (data not shown).

STZ-IMT504 rats presented a marked increase in NGN3positive islets and in NGN3-positive nuclei per islet,

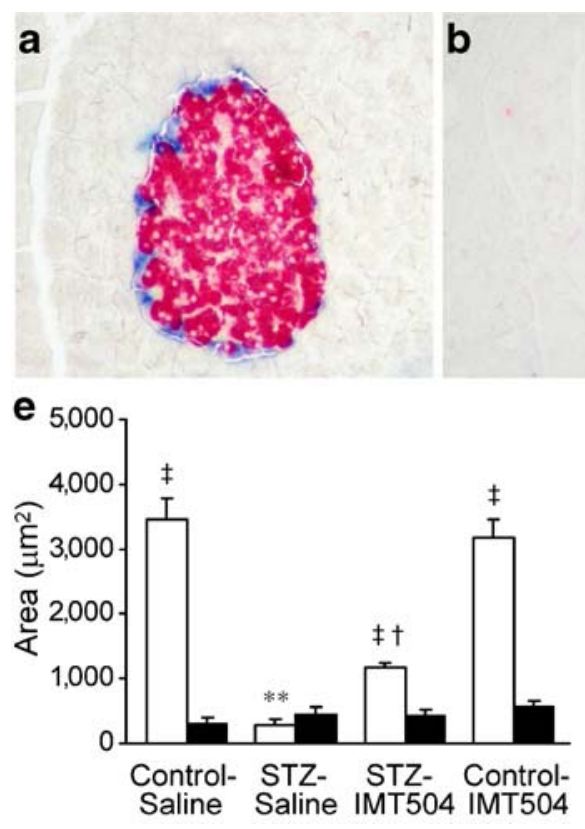

Fig. 2 Representative images of pancreatic islets from Control-Saline (a), STZ-Saline (b), STZ-IMT504 (c) and Control-IMT504 (d) immunostained for insulin (red) and glucagon (blue); magnification $\times 100$. e Insulin-positive (white bars) and glucagon-positive (black bars) areas. Two-way ANOVA interaction, $p<0.001$. Newman-Keuls test: $* *$ different from insulin areas in all other groups $(p<0.01),{ }^{\dagger}$ different
C

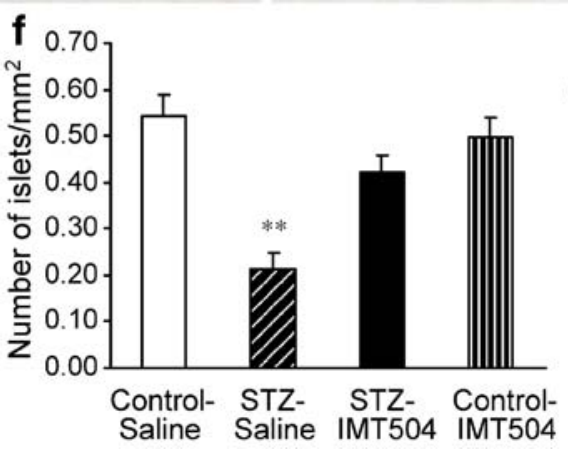

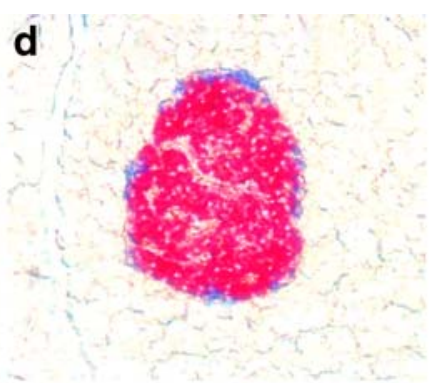

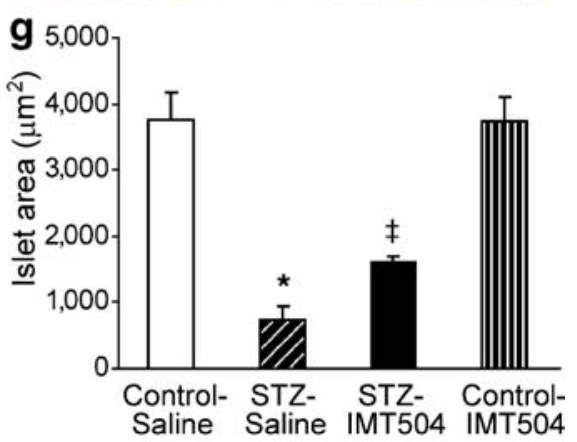

from glucagon areas in all other groups $(p<0.01)$, ${ }^{\ddagger}$ different from glucagon-positive area in each group $(p<0.01)$. f Number of islets per $\mathrm{mm}^{2}$ of pancreatic sections. One-way ANOVA, $p<0.01$. NewmanKeuls test: $* *$ different from all $(p<0.01)$. g Islet area in pancreatic sections, one-way ANOVA $p<0.001$. Newman-Keuls test: *different from all ( $p<0.05$ or better), ${ }^{\star}$ different from all $(p<0.05$ or better) 

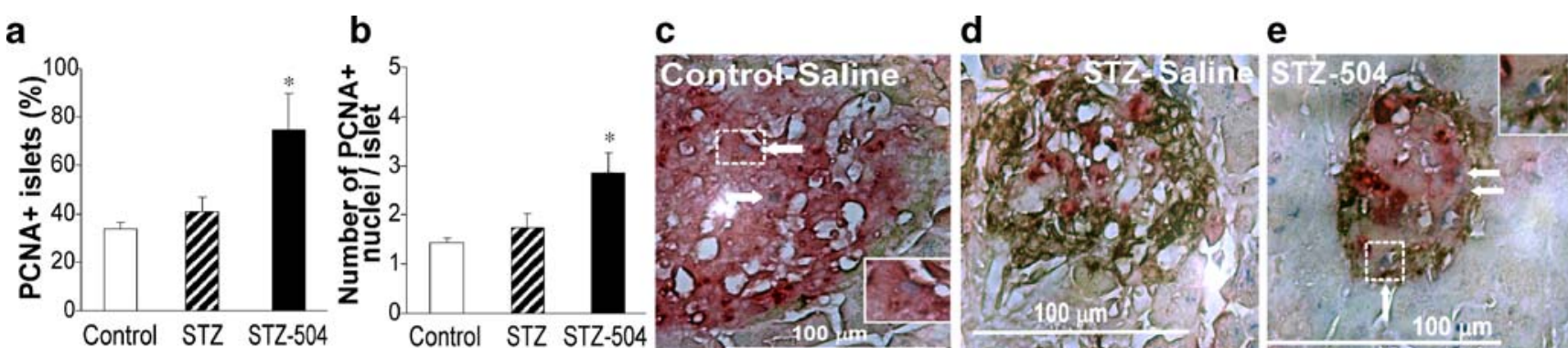

f

g

h
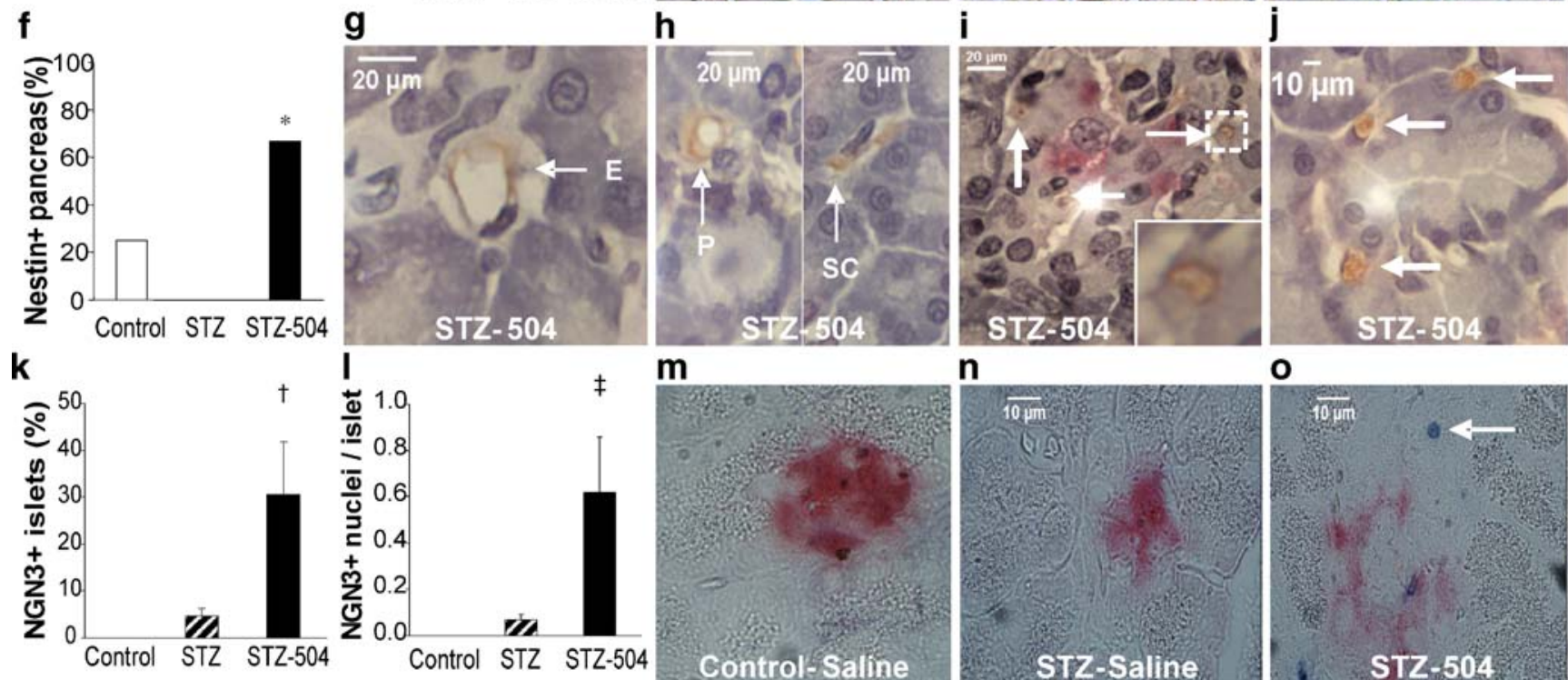

m

n

o
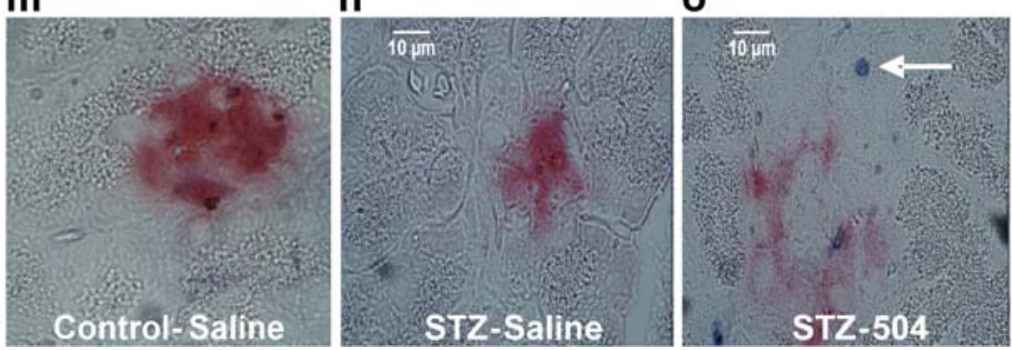

Fig. 3 IMT504 induced early PCNA (a-e), nestin (f-j) and NGN3 $(\mathbf{k}-\mathbf{o})$ staining in STZ-IMT504 rats. Control-Saline $(n=4)$, STZ-Saline $(n=6)$ and STZ-IMT504 $(n=6)$. a-e Triple staining for insulin (red), glucagon (brown) and PCNA (blue). a PCNA-positive islets as percentage in Control-Saline ( $n=126$ islets), STZ-Saline $(n=58$ islets) and STZ-IMT504 ( $n=51$ islets) rats. One-way ANOVA, $p<$ 0.02 . Tukey HSD test for unequal $n$ : *different from Control-Saline $(p<0.01)$ and from STZ-Saline $(p<0.02)$. b Number of PCNApositive nuclei per islet. One-way ANOVA, $p<0.03$. Tukey HSD test for unequal $n$ : *different from Control-Saline $(p<0.05)$. c Representative islet of Control-Saline; dotted-line square: one PCNA-positive nucleus pointed by arrow, inset: detailed amplification of dotted square. d Representative islet of STZ-Saline. e Representative islet of STZ-IMT504; arrows point to PCNA-positive nuclei; dotted-line square shows one PCNA-positive nucleus, amplified in the inset. $\mathbf{f}-\mathbf{j}$ Double staining for insulin (red) and nestin (brown). f Nestin-positive pancreases as percentage. $\chi^{2}$ test: * significantly different from STZSaline $(p<0.05)$. g Nestin-positive endothelium (E: arrow) of an STZ-
IMT504 rat. h Left, nestin-positive pericyte (P: arrow) surrounding an arteriole; right, nestin-positive stellate cell (SC: arrow) in pancreatic parenchyma of an STZ-IMT504 pancreas. i Representative islet of an STZ-IMT504 pancreas; arrows show nestin-positive microvasculature, dotted-lined square: microvasculature, inset: detailed amplification of dotted square. $\mathbf{j}$ Nestin-positive cells in exocrine acini (arrows) of an STZ-IMT504 pancreas. k-o Double staining for insulin (red) and NGN3 (blue). k NGN3-positive islets as percentage in Control-Saline ( $n=115$ islets), STZ-Saline ( $n=68$ islets) and STZ-IMT504 $(n=67$ islets) rats. One-way ANOVA, $p<0.02$. Tukey HSD test for unequal $n$ : $\dagger$ different from Control-Saline $(p<0.03)$ and STZ-Saline $(p<0.04)$. I Number of NGN3-positive nuclei per islet. One-way ANOVA, $p<$ 0.02 . Tukey HSD test for unequal $n$ : ${ }^{\star}$ different from Control-Saline $(p<0.04)$ and STZ-Saline $(p<0.04)$. m Representative islet of a Control-Saline pancreas. $\mathbf{n}$ representative islet of an STZ-Saline pancreas. o representative islet of an STZ-IMT504 pancreas, arrow pointing to an NGN3-positive nucleus significantly different from Control-Saline and STZ-Saline rats $(p<0.02)$. Control islets were NGN3-negative (Fig. $3 \mathrm{k}, 1)$. NGN3 was also observed in some duct and exocrine cells in STZ-Saline and STZ-IMT504 rats (not shown).

\section{Discussion}

IMT504 markedly reversed diabetes in STZ animals. A striking recovery of islet structure, number and cell composition was observed. The short time necessary to lower high blood glucose in STZ-IMT504 rats suggests an initial effect on pre-existing islet recovery and a possible later effect on islet neogenesis. An early increase in beta cell proliferation and in insulin secretion also point to islet recovery.

IMT504 induced the rapid appearance of tissue repair markers such as PCNA, CD31, nestin and NGN3. PCNA increased in STZ-IMT504 islets, indicating active proliferation, mainly in beta cells. Nestin, a non-endocrine lineage 
PPCM, was increased by IMT504 treatment in endothelial cells, pericytes and stellate cells, marking active regeneration [7] and angiogenesis [8]; this last variable was also indicated by increased CD31 in blood vessels. NGN3, an unambiguous marker for islet progenitors, was absent in normal islets and markedly increased in STZ-IMT504 rats, suggesting that IMT504 may be triggering NGN3 synthesis in pancreas-resident progenitor cells [9], similar to what was observed when administrating bone marrow-derived cells [10]. Our results are consistent with the view that the adult pancreas retains the potential to reactivate its embryonic mode of beta cell formation under the proper stimuli [11], and this capacity is greatly enhanced by IMT504. Nevertheless, progenitor recruitment from other compartments may also occur. Lineage-tracing studies would provide important information in this regard $[9,10]$.

Immunomodulatory actions of IMT504 must also be taken into consideration when analysing diabetes improvement. In this regard, microbial products such as Complete Freund's Adjuvant or Toll-like receptor agonists are known to have dissimilar effects on diabetes depending on the model $[1,2]$. No studies with PyNTTTTGT ODNs and autoimmune diseases have been developed so far. Therefore, the participation of the immune system in the IMT504induced rescue from diabetes in this model remains to be evaluated, as IMT504 may also be inducing cytokines, which may in turn induce the expression of PPCMs (nestin and NGN3) and/or preserving remaining beta cells.

In summary, IMT504 induced the recovery of pathognomonic signs of diabetes in rats and promoted the early production of tissue repair markers, such as PCNA, CD31, nestin and NGN3. These results point to IMT504 as an interesting candidate for future treatment of diabetes.

Acknowledgements This work was supported by grants from Immunotech, Argentina; CONICET, Argentina, PIP 5540; ANPCyT, Argentina, BID 1728/OC-AR PICT 2004 5-26307 to C. Libertun and BID 1728/OC-AR PICT 2006 No. 00200 to V. A. Lux-Lantos and UBA-ME 038. We thank E. Arany, Lawson Health Research Institute, London, ON, Canada, for the gift of monoclonal antibodies to NGN3 and nestin. F25A1B3 (NGN3) was developed by O. D. Masden and Rat-401 (nestin) was developed by S. Hockfield, Developmental Studies Hybridoma Bank (DSHB), under the auspices of the NICH and maintained by the University of Iowa, Iowa City, IA, USA. We also thank H. García-Rivelo, Hospital Italiano-Buenos Aires, for invaluable help in histological interpretation and C. Mirabelli (Gemma Biotech, Buenos Aires, Argentina) for technical assistance.

Duality of interest J. Zorzopulos is Vice-President and Stock owner of Immunotech, a for-profit organisation engaged in the development of biomedical products. The other authors declare that there is no duality of interest associated with this manuscript.

\section{References}

1. Nikoopour E, Schwartz JA, Singh B (2008) Therapeutic benefits of regulating inflammation in autoimmunity. Inflamm Allergy Drug Targets 7:203-210

2. Fallarino F, Volpi C, Zelante T et al (2009) IDO mediates TLR9driven protection from experimental autoimmune diabetes. J Immunol 183:6303-6312

3. Elias F, Flo J, Lopez RA, Zorzopulos J, Montaner A, Rodriguez JM (2003) Strong cytosine-guanosine-independent immunostimulation in humans and other primates by synthetic oligodeoxynucleotides with PyNTTTTGT motifs. J Immunol 171:3697-3704

4. Krieg AM, Yi AK, Matson S et al (1995) CpG motifs in bacterial DNA trigger direct B-cell activation. Nature 374:546-549

5. Hernando IA, Montaner AD, Rodriguez JM et al (2007) IMT504, the prototype of the immunostimulatory oligonucleotides of the PyNTTTTGT class, increases the number of progenitors of mesenchymal stem cells both in vitro and in vivo: potential use in tissue repair therapy. Stem Cells 25:1047-1054

6. Su SC, Pei D, Hsieh CH, Hsiao FC, Wu CZ, Hung YJ (2010) Circulating pro-inflammatory cytokines and adiponectin in young men with type 2 diabetes. Acta Diabetol. doi:10.1007/s00592009-0171-y

7. Crisan M, Yap S, Casteilla L et al (2008) A perivascular origin for mesenchymal stem cells in multiple human organs. Cell Stem Cell 3:301-313

8. Lardon J, Rooman I, Bouwens L (2002) Nestin expression in pancreatic stellate cells and angiogenic endothelial cells. Histochem Cell Biol 117:535-540

9. Xu X, D'Hoker J, Stange G et al (2008) Beta cells can be generated from endogenous progenitors in injured adult mouse pancreas. Cell 132:197-207

10. Gao X, Song L, Shen K, Wang H, Niu W, Qin X (2008) Transplantation of bone marrow derived cells promotes pancreatic islet repair in diabetic mice. Biochem Biophys Res Commun 371:132-137

11. Hanley NA, Hanley KP, Miettinen PJ, Otonkoski T (2008) Weighing up beta-cell mass in mice and humans: self-renewal, progenitors or stem cells? Mol Cell Endocrinol 288:79-85 